\title{
Biorecognition in Organic Field Effect Transistors Biosensors: The Role of the Density of States of the Organic Semiconductor
}

\author{
Marcello Berto, Stefano Casalini, Michele Di Lauro, Simone L. Marasso, Matteo Cocuzza, \\ Denis Perrone, Marcello Pinti, Andrea Cossarizza, Candido F. Pirri, Daniel Simon, Magnus \\ Berggren, Francesco Zerbetto, Carlo A. Bortolotti and Fabio Biscarini
}

\section{Journal Article}

\section{Tweet}

N.B.: When citing this work, cite the original article.

Original Publication:

Marcello Berto, Stefano Casalini, Michele Di Lauro, Simone L. Marasso, Matteo Cocuzza, Denis Perrone, Marcello Pinti, Andrea Cossarizza, Candido F. Pirri, Daniel Simon, Magnus Berggren, Francesco Zerbetto, Carlo A. Bortolotti and Fabio Biscarini, Biorecognition in Organic Field Effect Transistors Biosensors: The Role of the Density of States of the Organic Semiconductor, ANALYTICAL CHEMISTRY, 2016. 88(24), pp.12330-12338.

http://dx.doi.org/10.1021/acs.analchem.6b03522

Copyright: American Chemical Society http://pubs.acs.org/

Postprint available at: Linköping University Electronic Press

http://urn.kb.se/resolve?urn=urn:nbn:se:liu:diva-134080 


\title{
Biorecognition in organic field effect transistors biosensors: the role of the density of states of the organic semiconductor
}

Marcello Berto ${ }^{a}$, Stefano Casalini ${ }^{a_{+}}$, Michele Di Lauro, ${ }^{a}$ Simone L. Marasso ${ }^{b, c}$, Matteo Cocuzza $^{b, c}$, Denis Perrone ${ }^{d}$, Marcello Pinti ${ }^{a}$, Andrea Cossarizza ${ }^{e}$, Candido F. Pirri ${ }^{b, d}$, Daniel T. Simonf, Magnus Berggrenf, Francesco Zerbetto ${ }^{g}$, Carlo A. Bortolotti ${ }^{a^{*}}$ and Fabio Biscarini ${ }^{a}$ ${ }^{a}$ Dipartimento di Scienze della Vita, Università di Modena and Reggio Emilia, Via Campi 103, 41125 Modena, Italy;

${ }^{\mathrm{b}}$ Dipartimento di Scienza Applicata e Tecnologia (DISAT) - Politecnico di Torino, C.so Duca degli Abruzzi 24, 10129 Torino, Italy;

'IMEM-CNR, Parco Area delle Scienze 37, 43124 Parma, Italy;

dIstituto Italiano di Tecnologia, Center for Sustainable Futures, Corso Trento 21, 10129 Torino, Italy;

eDipartimento di Scienze Mediche e Chirurgiche Materno-Infantili e dell’Adulto, Università di Modena and Reggio Emilia, Via Campi 287, 41125 Modena, Italy;

fLaboratory of Organic Electronics, Department of Science and Technology, ITN, Linköping University, S-601 74 Norrköping, Sweden;

'Dipartimento di Chimica “G. Ciamician”, Università di Bologna, Via Selmi 2, 40126 Bologna Italy.

+Present address: Institut de Ciéncia de Materials de Barcelona (ICMAB-CSIC), 08193 Bellaterra, Spain.

\section{*Address correspondence to:}

Dr. Carlo Augusto Bortolotti, Dipartimento di Scienze della Vita, Università di Modena e Reggio Emilia, Via Campi 103, 41125 Modena, Italy; email:carloaugusto.bortolotti@unimore.it Tel: +390592058608

\begin{abstract}
Biorecognition is a central event in biological processes in the living systems that is also widely exploited in technological and health applications. We demonstrate that the Electrolyte Gated Field Effect Transistor (EGOFET) is an ultrasensitive and specific device that allows us to quantitatively assess the thermodynamics of biomolecular recognition between a human antibody and its antigen, viz. the inflammatory cytokine TNF $\alpha$, at solid/liquid interface. The EGOFET biosensor exhibits a super-exponential response at TNF $\alpha$ concentration below $1 \mathrm{nM}$ with a minimum detection level of $100 \mathrm{pM}$. The sensitivity of the device depends on the analyte concentration, reaching a maximum in the range of clinically-relevant TNF $\alpha$
\end{abstract}


1concentrations when the EGOFET is operated in the sub-threshold regime. At concentrations greater than $1 \mathrm{nM}$ the response scales linearly with the concentration. The sensitivity and the dynamic range are both modulated by the gate voltage. These results are explained by establishing the correlation between the sensitivity and the density of states (DOS) of the organic semiconductor. Then, the super-exponential response arises from the energydependence of the tail of the DOS of the HOMO level. From the gate voltage-dependent response, we extract the binding constant, as well as the changes of the surface charge and the effective capacitance accompanying biorecognition at the electrode surface. Finally, we demonstrate the detection of TNF $\alpha$ in human-plasma derived samples, as an example for point-of-care application.

Keywords: immune-sensor, organic bioelectronics, TNF $\alpha$, density of states, binding constant

\section{INTRODUCTION}

Biorecognition is a central phenomenon in living matter that relies on complex patterns of labile, non-covalent intermolecular interactions. It may involve small as well as very large biomolecules. In a technological context, it is exploited in self-organisation and large area patterning, as well as in the fabrication of diagnostics devices. In health applications, biorecognition is the paradigm on which pharmaceuticals operate, and leveraging biorecognition is crucial to the development of personalized medicine. At the experimental level, probing biorecognition is complicated and time consuming, as it requires ultra-sensitive techniques, often based on labels, that cannot readily be transferred outside specialized analytical laboratories. Label-free biorecognition detectors could thus fill the needs of a wide range of applications. While such detectors can be made ultra-sensitive and fabricated in large numbers at low cost, they are not widespread, especially in clinical applications. Electronic biosensors based on MOSFET devices were pioneered a few decades ago ${ }^{1-4}$. Also, silicon nanowire transistors and carbon-allotrope devices were proposed for ultra-sensitive detection $^{5,6}$. Organic field effect transistors are emerging as a promising platform for ultrasensitive detection of biological molecules ${ }^{7-10}$. In particular, Electrolyte Gated Field Effect Transistors (EGOFETs) directly couple the organic semiconductor (OSC) to the gate electrode throught the high capacitance of the electrolyte. EGOFET biosensors comprising immobilization of a specific receptor either on the $\mathrm{OSC}^{11}$ or on the gate $(\mathrm{G})$ electrode ${ }^{12-14}$ were demonstrated with sensitvity comparable to (and in some cases even better than) stateof-the-art techniques such as enzyme-linked immunosorbent assay (ELISA) or surface 
plasmon resonance (SPR). Significant advances have been made in the elucidation of the physical determinants of device response ${ }^{11,14}$, as well as in the control of the receptor orientation at the solid/liquid interface to optimize the sensing performances ${ }^{13,15}$. However, a more complete understanding of biorecognition and its coupling to the electronic channel, and the consequent control on both selectivity and sensitivity, is still lacking. There are a few reports of selective detection of antagonist analytes with label-free OFET biosensors ${ }^{7,10,14,16}$. It is assumed that the response of the biosensor should be linear vs concentration, but dose curves often deviate from this trend, especially at lower concentrations. The origin of this observation has not been studied.

In this work, we demonstrate the ultrasensitive detection (100 pM minimum detection level) of the primary inflammatory cytokine TNF $\alpha$ by means of an EGOFET immune-sensor with antibody-functionalized gate integrated in a microfluidic device. Our biosensor exhibits a super-exponential response at TNF $\alpha$ concentrations below $1 \mathrm{nM}$ and a linear response at larger concentrations. The sensitivity is maximum in the range of clinically-relevant concentrations and can be enhanced by operating the EGOFET in the sub-threshold regime. At larger concentrations, the sensitivity can be modulated up to $30 \%$ by driving the device in the saturation regime. In order to extract robust descriptors, we fit our data with a functional describing the concurrence of cooperative, Hill-like response at lower concentrations and linear Langmuir-like adsorption at the higher concentrations explored in this experiment. Both concentration-dependent sensitivity and the crossover between the two regimes are explained by establishing the correlation between the electrochemical potential, that increases upon TNF $\alpha$ recognition, and the consequent decrease of the positive charge carrier density. The latter is reconducted to, and explained by means of, the integral of the DOS of the HOMO level of the organic semiconductor. The extracted parameters demonstrate that our devices are extremely specific and provide a reliable estimate of the antibody/antigen binding constant. We finally demonstrate that our EGOFET selectively detects and quantifies TNF $\alpha$ in a cell medium where monocytes from human blood have been cultured and stimulated to produce this proinflammatory cytokine. 


\section{EXPERIMENTAL SECTION}

\section{Gate fabrication}

Silicon dies coated with a thin layer of gold on both sides were used as gate electrodes. The 4 mm x 4 mm Si dies were obtained from a 4" wafer (with a thickness of 525 +/-25 um, Single Side Polished). This wafer was strongly As doped (n-type), with a resistivity ranging from 1 to $6 \mathrm{mOhm} \bullet \mathrm{cm}$. The wafer was dipped in a HF (49\%):H2O solution (1:10 vol.), then rinsed several times in deionised water, in order to remove the native SiO2 layer. Gold (50 nm thick) was deposited on the polished side of the Si wafer using an electron beam evaporator (ULVAC EBX14D). A $5 \mathrm{~nm}$ thick Cr film was deposited as adhesion layer between $\mathrm{SiO} 2$ and the Au layer. The Cr layers were grown at a rate of $0.2 \mathrm{~nm} / \mathrm{s}$, and the deposition rate of the $\mathrm{Au}$ layers was kept constant at $0.3 \mathrm{~nm} / \mathrm{s}$. After the $\mathrm{Cr} / \mathrm{Au}$ deposition, the polished side of the wafer was coated with a 1.3um thick protective layer of AZ5214E photoresist (from Microchemicals $\mathrm{GmbH}$ ), dipped again in the $\mathrm{HF}$ (49\%): $\mathrm{H}_{2} \mathrm{O}$ solution (1:10 vol.) to remove the native oxide on the back side and rinsed several times in deionised water. Then, the $\mathrm{Cr}(5 \mathrm{~nm}) / \mathrm{Au}(50 \mathrm{~nm})$ layers were electron beam evaporated also on the wafer back side. The wafer was then diced in $4 \mathrm{~mm} \times 4 \mathrm{~mm}$ chips by means of a diamond dicing saw, and the protective photoresist on the polished side was stripped using acetone and ethanol as solvents.

\section{Electrical characterization}

All electrical measurements have been acquired in a buffer solution (PBS $50 \mathrm{mM}$, pH 7.4) in order to mimic physiological conditions. The buffer solution has been confined in a PDMS microfluidic, as shown in figure $1 \mathrm{~b}$. Source, drain and gate electrodes were connected to a Agilent B2902A Source Meter Unit. All measurements were carried out in atmosphere conditions (temperature and humidity). The I-V transfer characteristics were performed by sweeping the gate-source voltage $\left(\mathrm{V}_{\mathrm{GS}}\right)$ from $-0.2 \mathrm{~V}$ to $-0.8 \mathrm{~V}$ while leaving the drain-source voltage $\left(\mathrm{V}_{\mathrm{DS}}\right)$ constant at $-0.2 \mathrm{~V}$ (linear regime). Electrical characterization was done by means of a sample holder fabricated by CNC milling: the sample holder was composed by two polycarbonate supports modified for the electrical connections and clamped in order to firmly connect test pattern, microfluidic and gate electrode. Mesurements in complex matrices (i.e. supernatant from monocytes culture and stimulation) have been done by means a slightly different experimental setup, as described in refs 12 and 13: in these experiments the gate 
electrode was a polycrystalline Au wire functionalized as previously described, and immersed in a droplet of the supernatant confined by a PDMS pool..

\section{PDMS microfluidic fabrication}

PolyDiMethylSiloxane (PDMS) microfluidics were fabricated by casting onto a PolyMethylMethAcrylate (PMMA) mould obtained through a computer numerical control (CNC) milling machine. An array of 4 PDMS chips were obtained by a single casting process. Three inserts were mounted in the mould before the casting process in order to obtain the final inlet and outlet ports and the opening for the upper electrode. PDMS prepolymer and curing agent were thoroughly mixed (using a 10:1 in weight ratio) and successively degassed under vacuum for $5 \mathrm{~min}$, in order to avoid the presence of trapped air bubbles. The degassed PDMS mixture was then poured into the moulds, cured in a convection oven for $30 \mathrm{~min}$ at $90^{\circ} \mathrm{C}$ and subsequently peeled off from the master. Microfluidics chips of $5 \mu \mathrm{L}$ volume were finally obtained.

\section{RESULTS AND DISCUSSION}

\section{Transfer and dose curves}

We fabricated EGOFETs in the configuration schematically depicted in fig. 1a. The gate electrode (inset of fig. 1a) was the sensing element Its functionalization with the anti-TNF $\alpha$ antibody was performed according to the protocol described in ref. 13. The electrolyte solution was Phosphate Buffer Saline (PBS) at pH 7.4. The EGOFET channel consisted of a $15 \mathrm{~nm}$ thick pentacene film grown by high vacuum sublimation. The device was then integrated with a microfluidic system (fig. 1b) in order to confine the solution on the active area. The microfluidics guaranteed a sealed environment capable of keeping the electrolyte volume constant and avoiding contamination from adventitious agents during the electrical characterization. The same microfluidics setup was used to functionalize the gate electrode in situ. 

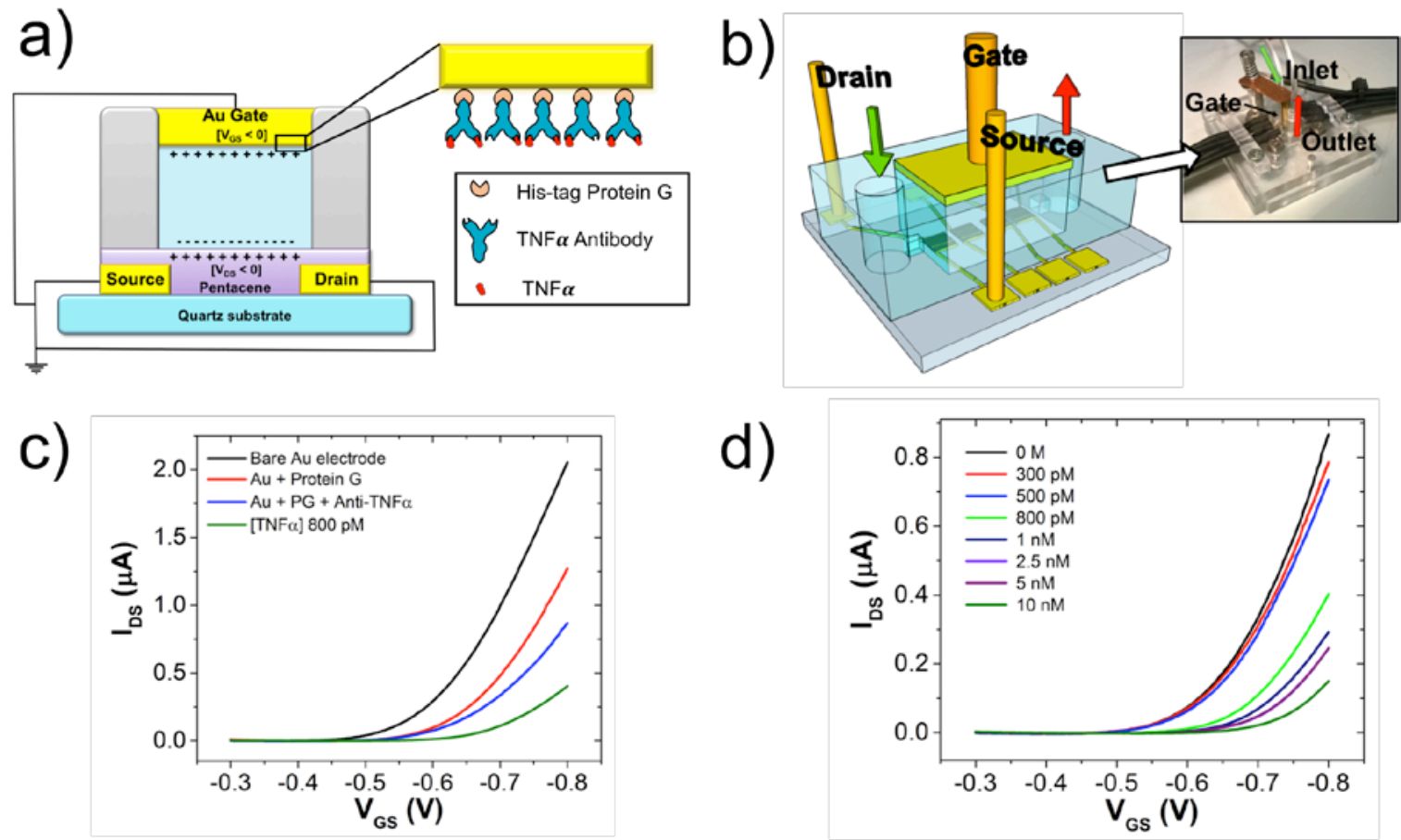

d)

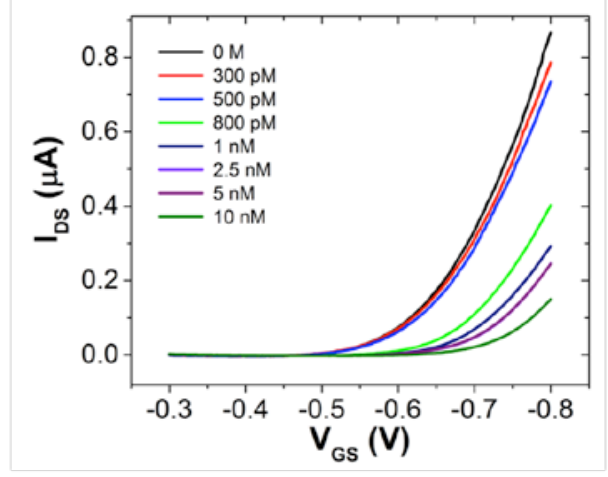

e)

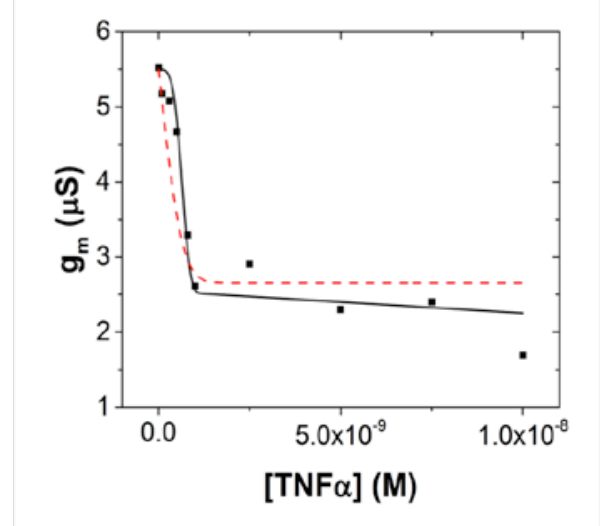

f)

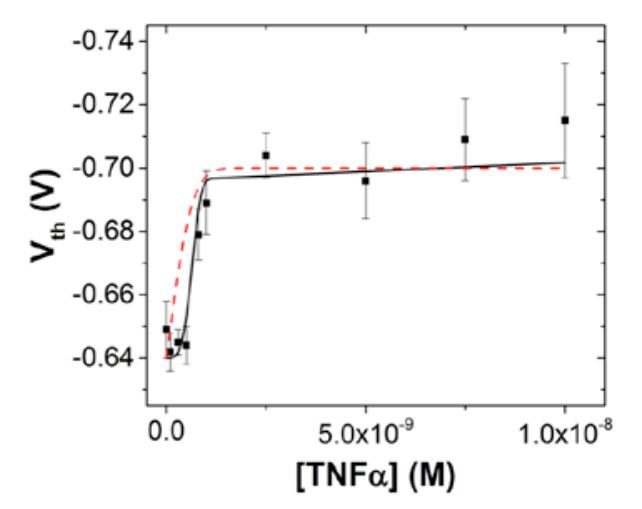

Figure 1. a) Schematics of the EGOFET device with a zoom of the functionalized gate. b) Schematic drawing of the microfluidics and a photo of the experimental setup comprising the electrical connections and fluidic inlets. c) Transfer curves recorded at each functionalization step. d) Transfer characteristics of a pentacene EGOFET upon exposure to TNF $\alpha$ solutions in PBS buffer. The corresponding TNF $\alpha$ concentration is reported in the legend. e) Transconductance and $f$ ) threshold voltage trends as a function of TNF $\alpha$ concentration. Solid black and dashed red lines are fits using the functional in eq. 1 and an error function (erf), respectively.

Fig. 1c shows the evolution of the transfer characteristics during the different functionalization steps. As from previous reports, ${ }^{12,13,17}$ changes are observed upon each step. Notably, a significant change in the transfer curve was observed upon exposure of the device to an aqueous solution containing $800 \mathrm{pM} \mathrm{TNF} \alpha$. The leakage current was always lower than $50 \mathrm{nA}$. This finding, along with an almost negligible hysteresis, indicates that no electrochemical doping of the pentacene thin film occured. Fig. 1d shows representative 
transfer characteristics ( $I_{D S}$ vs $V_{G S}$ at $V_{D S}=-0.2 \mathrm{~V}$ ) for selected concentrations of TNF $\alpha$. The trend observed upon exposure of the device to TNF $\alpha$ buffer solutions indicates a decrease of current upon adsorption of the cytokine on the gate. Specifically, as TNF $\alpha$ concentration increases i) the transfer curves shift to lower current values; ii) the slope, viz. the transconductance $g_{m}$ (fig. 1e), of the linear region decreases; iii) the threshold voltage, $V_{t h}$ (fig. 1f) shifts to more negative values. .This evidence points to the mechanism of coupling between the binding of the antigen and the carriers in the channel. The negative shift of the threshold voltage indicates that additional traps for charge carriers are created upon TNF $\alpha$ binding. At physiological $\mathrm{pH}$ the charge of TNF $\alpha$ is slightly negative, being its isoelectric point is 6.44. The correlated trends of $g_{m}$ and $V_{t h}$ suggest that the adsorption of TNF $\alpha$ decreases the charge-carrier density. Both data sets can be fitted starting from the functional defined below in eq. 1 , as explained later.

To quantitatively assess the response of the device, we define the device response signal $S=-$ $\Delta I / I_{0}$ as the relative current variation normalized to the transfer curve value for $[\mathrm{TNF} \alpha]=0 \mathrm{M}$. The dose curve, $S$ vs $[\mathrm{TNF} \alpha]$ in the range from $100 \mathrm{pM}$ to $10 \mathrm{nM}$, was extracted at each gate voltage $V_{G S}<-0.6 \mathrm{~V}$. In fig. 2a we plot a representative dose curve $S$ vs [TNF $\alpha$ ], while in fig. $2 \mathrm{~b}$ we plot the curves recorded at selected gate voltages. 


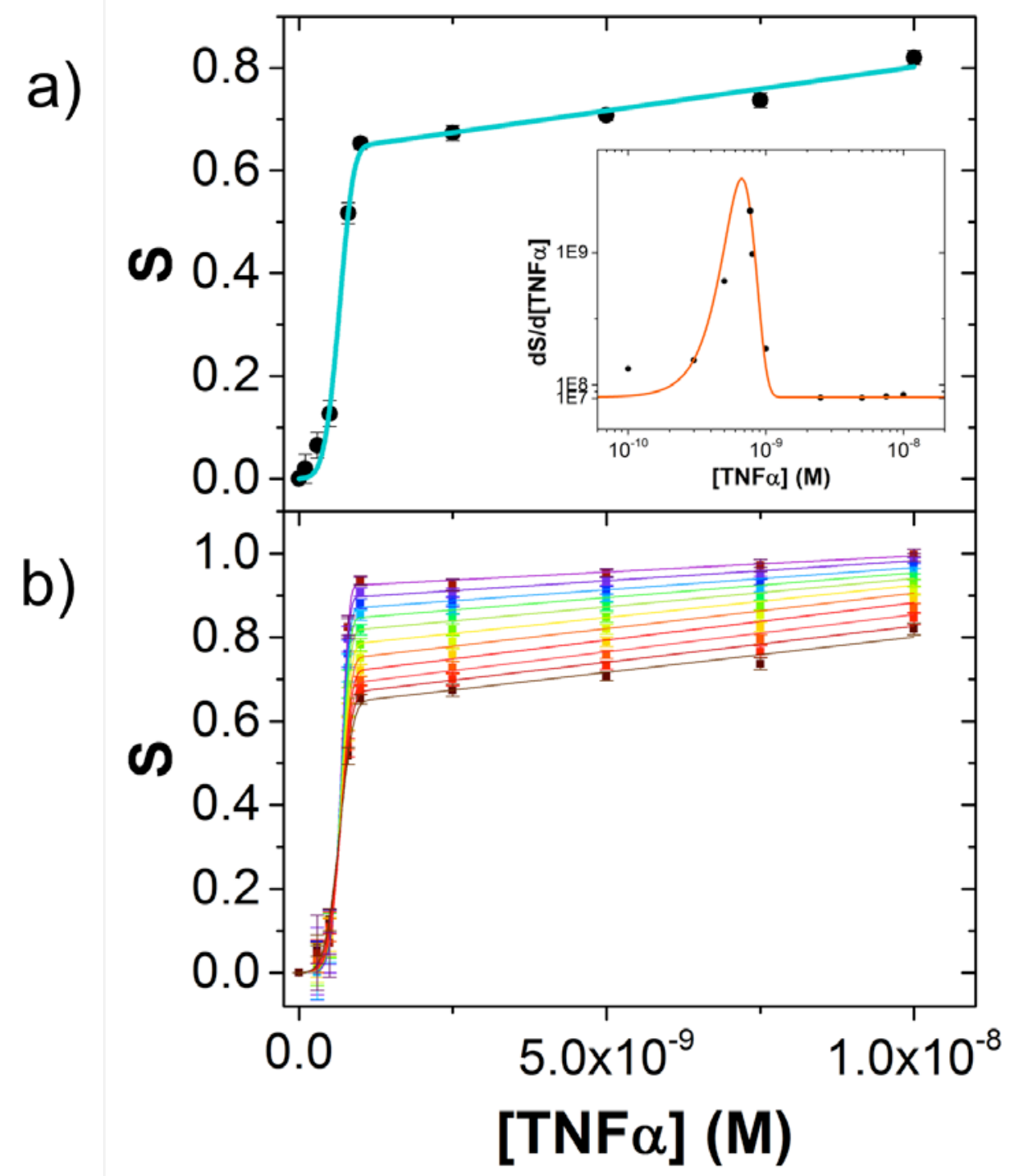

Figure 2. a) Dose curve $S$, acquired at $V_{G S}=-0.8 \mathrm{~V}$, vs molar concentration of $T N F \alpha$. The error bars correspond to the rms of $S$ averaged over four devices measured from two different test patterns. Continuous line is the best-fit with functional eq. 1. Inset: sensitivity estimated as the numerical derivative of $S$ data. The continuous line is the derivative of the functional eq. 1 calculated with best-fit parameters of the dose curve in 2a; b) Dose curves acquired at decreasing gate voltages from $V_{G s}=-0.6 \mathrm{~V}$ (top curve,

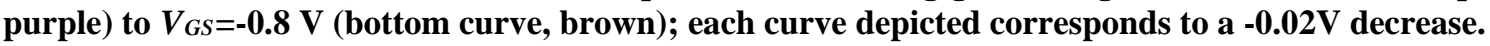

The dose curve exhibits a monotonic trend as a function of [TNF $\alpha$ ] (see fig. 2a); depending on the cytokine concentration range, two distinct response regimes are identified. At [TNF $\alpha$ ] $<1 \mathrm{nM}$, a steep increase of the dose curve occurs. For [TNF $\alpha$ ] about $1 \mathrm{nM}$, a second region with lower slope and a linear response sets in. Since the slope is the effective sensitivity of the sensor, we infer that the sensitivity of the immune-EGOFET changes in different concentration ranges. In particular, sensitivity is greater at the lowest [TNF $\alpha$ ]. In the inset of fig. 2a, we plot the sensitivity as the numerical derivative $d S / d[T N F \alpha]$ vs $[T N F \alpha]$. It is 
evident that the device responds in a wide range of analyte concentration, spanning two orders of magnitude of [TNF $\alpha]$. Most notably, the sensitivity increases by nearly one order of magnitude at the lower concentrations and peaks at $0.8 \mathrm{nM}$ concentration. For [TNF $\alpha]>0.8$ $\mathrm{nM}$, the sensitivity decreases dramatically (two orders of magnitude lower than the peak value). Therefore, the highest sensitivity falls in the concentration range $[\mathrm{TNF} \alpha]<1 \mathrm{nM}$. This optimal sensitivity range makes the immune-EGOFET well-suited for applications with real bodily fluids, since both physiological and pathological levels of this inflammatory biomarker usually lie below $\mathrm{nM}$ concentration. It also hints at an attractive feature of EGOFET biosensors: the response is "amplified" at the lowest concentrations ${ }^{12}$. The origin of this ultrahigh sensitivity will be discussed further below.

\section{Analysis of the dose curve}

We quantitatively assess the device response by extracting the relevant figures of merit from the experimental data. Upon investigating different functions (Gaussian, Langmuir and Hill functions) we devised the following functional to fit the dose curve:

$$
S=\frac{K_{a}\left\{c_{D O S}\left(1-e^{-\left(\frac{[T N F \alpha]}{\xi}\right)^{\gamma}}\right)+[T N F \alpha]\right\}}{1+K_{a}\left\{c_{D O S}\left(1-e^{-\left(\frac{[T N F \alpha]}{\xi}\right)^{\gamma}}\right)+[T N F \alpha]\right\}}
$$

With this function we obtain minimum chi-square values and better distributed residuals with respect to library functions. The first term in the numerator of eq. 1 accounts for the superlinear increase of the signal $S$ at the lower concentrations, while the second term accounts for the linear response at higher concentrations. At the lowest order, in case of an integer $\gamma$, eq. 1 yields a combination of a linear term and a power law:

(2) $S \approx K_{a}\left\{c_{D O S}\left(\frac{[T N F \alpha]}{\xi}\right)^{\gamma}+[T N F \alpha]\right\}$.

The constant $K_{a}$ is the binding constant of the antibody-antigen pair at the gate electrode. The coefficient $C D O S$, whose dimension is molar concentration, is a device parameter related to the tail of the density of states (DOS) of the organic semiconductor, described in more detail below. The product $K_{a} \cdot{ }^{\cdot}{ }_{D O S}=S_{\max }$ indicates the maximum value attained by $S$ in the lowconcentration region, and $\xi$ is the concentration that marks the crossover between the two regimes; $\gamma$ is directly related to how steeply the signal rises with the analyte concentration. As an example, a value $\gamma=2$ would turn the first term in bracket in eq. 1 into a complement to a Gaussian. It should be noted that eq. 1 yields a Langmuir adsorption isotherm when $c D O s<<1$, 
else Hill adsorption isotherm with exponent $\gamma$ when $c_{D O S}>>1$. Thus, eq. 1 links two limit regimes which are widely used to fit data obtained in similar experiments ${ }^{14,18}$.

The continuous lines in fig. $2 \mathrm{a}$ and $2 \mathrm{~b}$ are the best-fit with the function eq. 1 . The fit of the whole set of experimental data in fig. 2a yields $C_{D O S}=(3.8 \pm 0.7) \times 10^{-8} \mathrm{M}, \xi=(7.1 \pm 0.2) \times 10^{-10}$ $\mathrm{M}$, and $\gamma=(4.3 \pm 0.7)$. The best fit values show that more than $60 \%$ of signal variation is produced by the adsorption at concentrations smaller than $\xi$. The best-fit binding constant is $K_{a}=(1.7 \pm 0.1) \times 10^{7} \mathrm{M}^{-1}$. Interestingly, this value is comparable with reported antibody/antigene binding constant ${ }^{19-21}$, supporting the occurrence of specific binding because of the suitable orientation of the antibodies on the functionalised gate electrode.

The dose curve in fig.2a was obtained by plotting the signal $-\Delta I / I_{0}$ at $V_{G S}=-0.8 \mathrm{~V}$, while fig.2b displays the dose curves obtained for different gate voltages $V_{G S}$ ranging from $-0.6 \mathrm{~V}$ to $-0.8 \mathrm{~V}$. The actual number of dose curves acquired, and hence the dimension of the parameter set, matches the number of experimental points in each transfer curve, viz. 250, of which nearly 100 were falling in the on-regime of the transfer curve (i.e., $-0.8 \mathrm{~V}<V_{G S}<-0.6 \mathrm{~V}$ ). Fig. 3 displays the dependence of the fitting parameters $\gamma, c_{D O S}, S_{\max }$ and $K_{a}$ as a function of $V_{G S}$, corresponding to the fit in fig. $2 \mathrm{~b}$. 


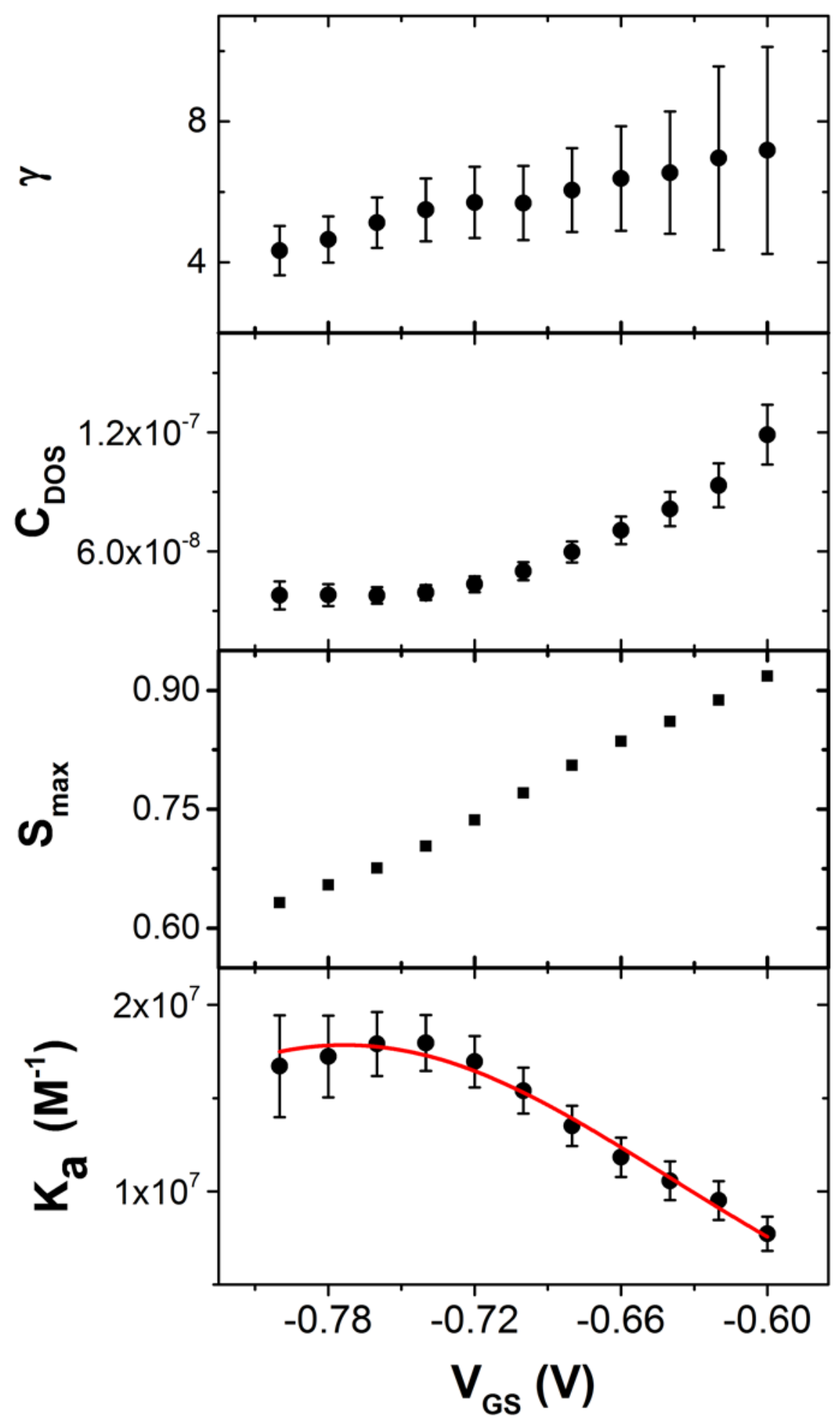

Figure 3. Dependence of the best-fit parameters from eq. 1 (described in the main text) vs gate voltage $V_{G S}$. Error bars are the estimated errors from the best fit of the dose curves. The solid red line in the bottom panel is the best fit with eq. 15.

As $V_{G S}$ increases (in magnitude), $\gamma$ decreases from 7 to $4, C D O S$ decreases then reaches a plateau around $V_{G S}=-0.72 \mathrm{~V}, S_{\max }$ decreases linearly from about $90 \%$ down to $60 \%$ and $K_{a}$ 
increases by about $50 \%$ of its value at $V_{G S}=-0.6 \mathrm{~V}$ reaching a maximum or a plateau at $V_{G S}<$ $-0.75 \mathrm{~V}$. The correlation among the mean values accompanied by an increase in the error bars with the increased mean value is due to a systematic error ascribed to the finite set of concentration points used for the best fit procedure.

Although the sensitivity is invariably larger at the lowest concentrations, it is apparent that the range of the dose curve is modulated by the gate voltage. The quasi-linear trend of the exponent $\gamma$, also paralleled by that of $S_{\max }$, clearly shows that smaller in magnitude (less negative) $V_{G S}$ values result in higher sensitivity in the sub-nM regime. This shows that, when aiming at sensing in the sub-nM range, the device performance can be maximized by working in the sub-threshold regime. Interestingly, the specificity, associated to the value of $K_{a}$, will be higher at more negative values of $V_{G S}$, and would thus be enhanced by operating the device in saturation regime.

\section{Understanding the EGOFET response}

The two regimes observed in fig. 2 are correlated to the trends exhibited by $g_{m}$ in fig. 1e and $V_{\text {th }}$ in fig. 1f. The vertical shift of the gate electrochemical potential upon adsorption shifts the electrochemical potential of the electrolyte and consequently re-adjusts the electrochemical potential $\mu$ of the organic semiconductor channel. The electrochemical potential $\mu$ would be the Fermi level $\mu_{0}$ at low $T$. In order to extract the essence of the mechanism, we assume that the concentration [TNF $\alpha$ ] linearly modulates $\mu$ by a potential energy shift $\phi$ : $\mu=$ $\mu_{0}+\phi=\mu_{0}+k \cdot[\mathrm{TNF} \alpha]$. The electronic structure of the gate electrode (which is also determined by the geometry of the electrodes, and by the areal density and orientation of the recognition group) enters here in the constant $k$ between $\phi$ and [TNF $\alpha$ ].

The signal $S$ is proportional to the change of conductance $\sigma$ of the EGOFET channel. The adsorption of TNF $\alpha$ leads to a decrease of the hole density, which implies that the Fermi level of the pentacene film increases. Based on this argument, it turns out that $\phi>0$. According to linear response theory ${ }^{22}$, the variation of $\sigma$ can be approximated as:

(3) $S[T N F \alpha] \propto \int_{\mu_{0}}^{\mu_{0}+\phi} D O S(E) d E=\int_{0}^{\phi} D O S\left(E+\mu_{0}\right) d E$

where $\operatorname{DOS}(E)$ is the density of states at energy $E$ of the organic semiconductor. The sensitivity will then be:

(4) $\frac{d S}{d[T N F \alpha]} \propto \frac{d}{d \phi} \int_{0}^{\phi} \operatorname{DOS}\left(E+\mu_{0}\right) d E=\operatorname{DOS}\left(\phi+\mu_{0}\right)$ 
The sensitivity of the EGOFET is proportional to the density of states of the organic semiconductor near the Fermi level. Thus, the inset of fig. 2a depicts the DOS of the pentacene: it is skewed and not gaussian.

To explain the rationale of our argument, we refer to the schematic drawing in fig. 4.
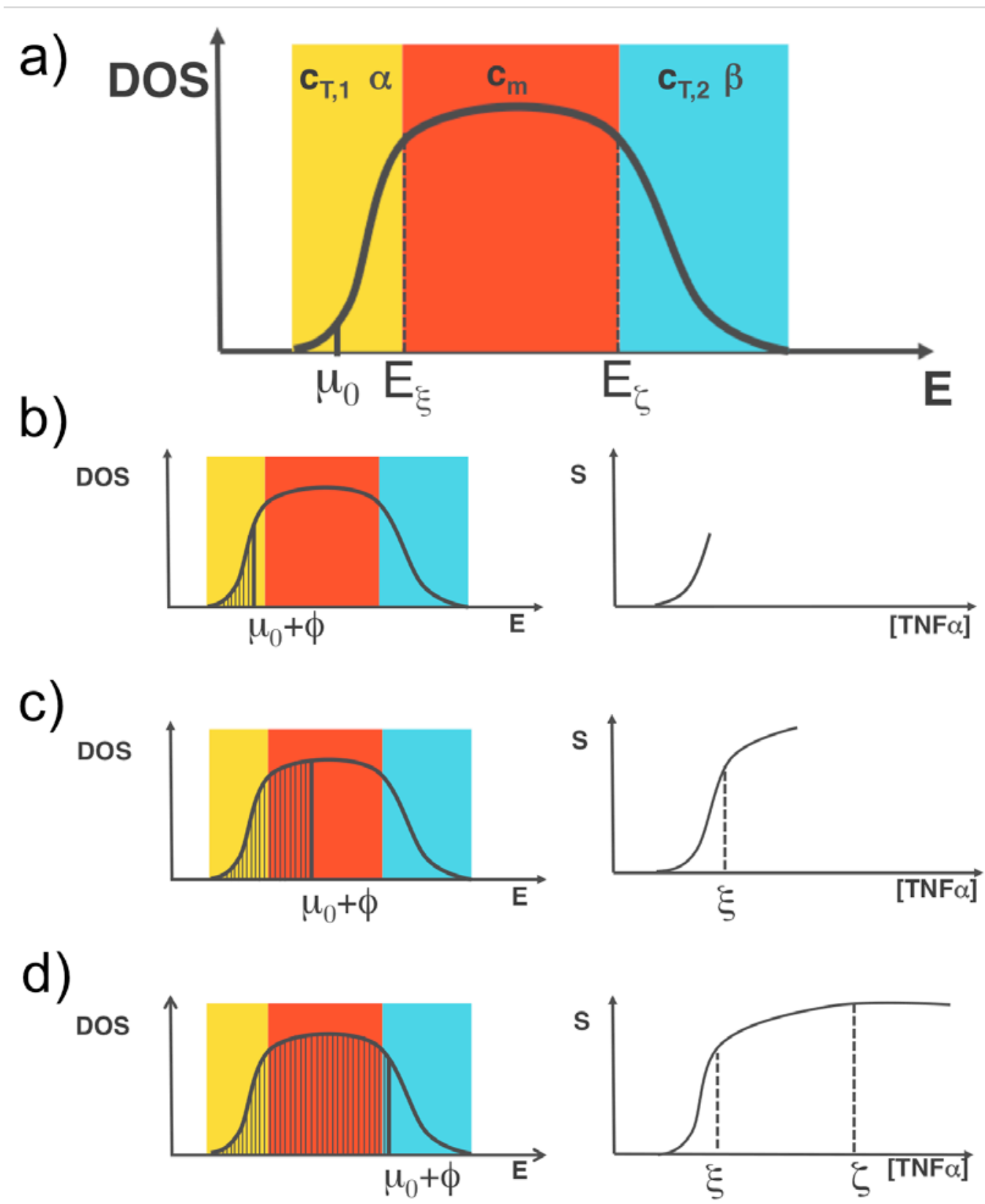

Figure 4. Schematic representation of the interplay between the voltage $\phi$ and the density of states (DOS) of pentacene HOMO. a) Model DOS(E) for a typical OSC. Three regions are higlighted: the yellow and blue background identify the rapidly varying DOS tails, whose shape is approximated as a power law with parameters $C_{T, 1}$ and $\alpha$, and $C_{T 21}$ and $\beta$, respectively. $E \xi$ and $E \zeta$ define the energy values separating the three regions and $\mu_{0}$ is the electrochemical potential of the OSC in the absence of adsorbed analyte. The central smooth region (red background) in the text is approximated by a constant $c_{m}$ (see eqs. 5 to 7).. b-d) The shape of the dose curve (right) mirrors the integral of the the DOS(E): for $[T N F \alpha]<\xi(b)$, the analyte adsorption causes a shift of $\mu_{0}$ to a $\mu_{0}+\phi$ value still in the low energy tail (yellow region): the signal rises steeply. (c) For $\xi<[T N F \alpha]<\zeta, \mu_{0}+\phi$ lies in the smooth region of DOS(E) (red region) and the signal rises 
smoothly. (d) For $[T N F \alpha]>\zeta, \mu_{0}+\phi$ falls in the upper energy tail (blue region) and the signal slows down or saturates.

The initial Fermi level $\mu_{0}$ lies in the lower energy tail of the HOMO DOS of pentacene. The increase of the upper integration limit vs the concentration makes the integration range larger, starting from the Fermi level. For sake of simplicity, we envision DOS(E) as a piecewise function rapidly varying at the tails and smoothly varying in the middle of the band (here we approximate the central region with a constant $c_{m}$, like in a 2D Fermi gas):

(5) $\operatorname{DOS}(E) \approx c_{T, 1}\left(E-\mu_{0}\right)^{\alpha}$ for $E \in\left[\mu_{0} ; E_{\xi}[\right.$

(6) $\operatorname{DOS}(E) \approx c_{m}$ for $E \in\left[E_{\xi} ; E_{\zeta}[\right.$

(7) $\operatorname{DOS}(E) \approx c_{m}-c_{T, 2}\left(E-E_{\zeta}\right)^{-\beta}$ for $E \in\left[E_{\xi} ; E_{\max }\right]$

In eq. 5 we illustrate the $\operatorname{DOS}(E)$ at the tails approximated as a power law, albeit any suitable functional, including the canonical gaussian one, would serve the purpose. Being the DOS a continuous function, eqs. 5-7 must take the same value at the limits of their respective ranges, thus leading to the equality:

(8) $c_{T, 1}\left(E_{\xi}-\mu_{0}\right)^{\alpha}=c_{T, 1}(k \cdot \xi)^{\alpha}=c_{m}$

which defines the proportionality constant $k$ in terms of the crossover concentration $\xi$ as:

(9) $k=\left(\frac{c_{m}}{c_{T, 1}}\right)^{\frac{1}{\alpha}} \xi^{-1}$

The value of $\xi$ can be extracted from the experimental data as in eq. 1 and 2. As TNF $\alpha$ is adsorbed at the gate, electrons fill progressively the (partially empty) HOMO states spreading over the energy interval $\left(E_{\max }-\mu_{0}\right)$ from $\mu_{0}$ to $\mu_{0}+\phi$. Because the band width of pentacene is of the order of $100 \mathrm{meV}$, or $10 \mathrm{kJmol}^{-123,24}, \phi$ can take up to $100 \mathrm{meV}$ as maximum value. The effective energy interval of the HOMO band accessible to the experiment depends on $\mu_{0}$, and hence on the gate voltage and the intrinsic doping level of the organic semiconductor.

We now integrate then eq. 3 by substituting eq. 5 in it, so to obtain:

$(10) S \approx c_{T, 1}(\alpha+1) \phi^{\alpha+1}=\left\{c_{T, 1}(\alpha+1)\left(\frac{c_{m}}{c_{T, 1}}\right)^{\frac{\alpha+1}{\alpha}}\right\}\left(\frac{[T N F \alpha]}{\xi}\right)^{\alpha+1}$ for $\phi \in\left[0 ; E_{\xi}-\mu_{0}[\right.$
$(11) S \approx c_{m} \phi=\left\{c_{m}\left(\frac{c_{m}}{c_{T, 1}}\right)^{\frac{1}{\alpha}}\right\} \frac{[T N F \alpha]}{\xi}$ for $\phi \in\left[E_{\xi}-\mu_{0} ; E_{\zeta}-\mu_{0}[\right.$
(12) $S \approx c_{m} \phi-c_{T, 2} \ln \left(\frac{\phi}{E_{\zeta}}\right)=\left\{c_{m}\left(\frac{c_{m}}{c_{T, 1}}\right)^{\frac{1}{\alpha}}\right\} \frac{[T N F \alpha]}{\xi}-c_{T, 2} \ln \left(\frac{[T N F \alpha]}{\zeta}\right)$ for $\left[E_{\zeta}-\mu_{0} ; E_{\max }-\mu_{0}\right]$ 
Equations 10 and 11 reproduce the trend of the dose curves shown in fig. 2, and described by eqs. 1 and 2. This is shown in fig. 3b and 3c. In particular, we recognize that the power law in eq. 10 maps the approximation in eq. 2 by equating $\gamma=\alpha+1$, while eq. 11 matches the linear regime. From the values reported in fig. 2c, with focus on the $\gamma$ decrease at more negative $V_{G S}$, we infer that the gate potential (or electric field) broadens the DOS tail, thus leading to trapping of the holes. We therefore conclude that both the exponent $\gamma$ and the coefficient $c$ DOS in eq. 1 and 2 depend on the pentacene DOS, and, specifically, on the energy variation of the tail compared to the smoothly-varying region.

We also infer that the tail of the DOS is not gaussian, as usually assumed, instead varies more rapidly in energy and is narrower, approaching a delta function at the concentration $\xi$. Therefore, the integral of the DOS as in fig. 4a resembles a step function as the gate voltage $\mathrm{V}_{\mathrm{GS}}$ is the sub-threshold regime. By inspecting eq. 12, and looking at the schematics in fig. $4 \mathrm{~d}$, it is clear that the response vs [TNF $\alpha$ ] will not grow monotonically, instead will saturate above the concentration $\zeta$. This latter regime is not observed in our range of [TNF $\alpha$ ].

We therefore infer that the shape of the dose curve is determined by the tail of the DOS. To the best of our knowledge, only one paper describes an EGOFET-based biosensor with functionalization on the gate electrode ${ }^{14}$, while immobilization of the sensing moiety typically takes place at the OSC/electrolyte interface ${ }^{7,25}$. As a consequence of our ansatz, a smoother rise of the dose curve should be expected for an OSC more disordered than pentacene, or even amorphous, owing to the larger spreading of the tail of density of states. Moreover, our analysis suggests that it should be possible to use an EGOFET (bio)sensor as a tool to complement other methods $\mathrm{s}^{26-32}$ for the investigation of the DOS of different organic semiconductors.

To sum up, the signal $S$ of the EGOFET mirrors the integral of the DOS of the HOMO of OSC in an energy window modulated by the analyte concentration. The analyte adsorption at the gate shifts the Fermi level in the HOMO band, reducing the number of holes in it. As a consequence, the trend of $S$ vs $[\mathrm{TNF} \alpha]$ has to be paralleled by the trend of the transconductance $g_{m}$ and of $V_{t h}$ as a function of [TNF $\alpha$ ], since they arise from the decrease of the density of mobile carriers and from the increase of density of immobilized holes, repectively. This is confirmed by the solid lines in figs. 1e and 1f, which are the best fits to $g_{m}$ vs $[\mathrm{TNF} \alpha]$ and $V_{\text {th }}$ vs $[\mathrm{TNF} \alpha]$, respectively, with functional eq. 1. If the $D O S(E)$ were a gaussian function, then the signal $S$ and the correlated trends of $g_{m}$ and $V_{t h}$ would have 
followed an error function $\operatorname{erf}([\mathrm{TNF} \alpha] / \xi)^{33}$. The fitting of $S$ vs $[\mathrm{TNF} \alpha]$ with $\operatorname{erf}([\mathrm{TNF} \alpha] / \xi)$ provides less satisfactory results both for $g_{m}$ and $V_{t h}$ : the fits with the error function are comparable to those obtained with the function in eq. 1 at high concentrations, but deviate substantially in the sub-nM regime (see overlay of dashed red and solid black lines in figs. 1e and 1f).

\section{EGOFET to measure the thermodynamics of bio-recognition}

We finally discuss the trend of the binding constant $K_{a}$ as a function of $V_{G S}$. In the following we treat it as a thermodynamic dimensionless constant, thus abandoning the dimension $\mathrm{M}^{-1}$. In this framework, we recognize that $K_{a}$ can be factorized as:

(13) $K_{a}=e^{\left(-\frac{\Delta G_{0}}{R T}\right)} \cdot e^{\left(-\frac{\Delta G_{e}}{R T}\right)}$

where $\Delta G_{0}$ is the standard molar free energy of antibody-antigen binding at the neutral electrode interface ${ }^{14}, \Delta G_{e}$ is the molar electrostatic contribution to the free energy, $R$ is the gas constant, and $T$ is the absolute temperature. We expand the (enthalpic part of) molar electrostatic free energy $\Delta G_{e}$ at the $2^{\text {nd }}$ order as:

(14) $\Delta G_{e}=\delta Q_{m, e f f} \cdot\left(V_{G S}-V_{t h}\right)+\delta C_{m, e f f} \cdot\left(V_{G S}-V_{t h}\right)^{2}$

so that eq. (13) becomes

(15) $-R T \cdot \ln K_{a}=\Delta G_{0}+\delta Q_{m, e f f} \cdot\left(V_{G S}-V_{t h}\right)+\delta C_{m, e f f} \cdot\left(V_{G S}-V_{t h}\right)^{2}$

The linear term in eqs. $(14,15)$ represents the contribution due to the changes of the surface charge $\delta q_{s}$ and of the surface dipole moment $\delta \mu_{s}$ upon Ab-Ag binding. The quadratic term is due to the change of capacitance $\delta C_{i}$ at the interface between electrolyte and gate electrode and also includes the change of polarizability $\delta \alpha$.

We fit the $K_{a}$ values in fig. $3 d$ with eq. 15 , setting $V_{\text {th }}$ equal to its value at [TNF $\alpha$ ] $=0 \mathrm{M}$. The best fit parameters, with their estimated errors, are shown in Table 1.

Table 1. Best fit parameters for $K_{a}$ vs $V_{G S}$.

\begin{tabular}{|l|l|l|}
\hline Binding contribution & Charge contribution & Capacitive contribution \\
\hline$\Delta \mathrm{G}_{0} / \mathrm{RT}$ & $\delta \mathrm{Q}_{m, \text { eff }} / \mathrm{RT}\left(\mathrm{V}^{-1}\right)$ & $\delta C_{m, \text { eff }} / \mathrm{RT}\left(\mathrm{V}^{-2}\right)$ \\
\hline$-16.26 \pm 0.01$ & $7.0 \pm 0.4$ & $28.5 \pm 3.6$ \\
\hline$\Delta \mathrm{G}_{0}\left(\mathrm{~kJ} \cdot \mathrm{mol}^{-1}\right)$ & $\delta Q_{m, \text { eff }}\left(\mathrm{kC} \cdot \mathrm{mol}^{-1}\right)$ & $\delta C_{m, e f f}\left(\mathrm{kF} \cdot \mathrm{mol}^{-1}\right)$ \\
\hline$-40.27 \pm 0.02$ & $17.3 \pm 1.0$ & $70.6 \pm 8.9$ \\
\hline
\end{tabular}


We first notice that $\Delta G_{0}$ is of the order of $40 \mathrm{~kJ} \mathrm{~mol}^{-1}$, in agreement with values reported for other specific antibody-antigen standard molar free energy changes ${ }^{14}$. This value is lower than $\Delta G_{0}$ for antibody-antigen obtained in ref. ${ }^{13}$, however this is not surprising as the values of binding free energy changes extracted from force curves are known to be usually overestimated with respect to the values obtained through association/dissociation kinetics. In general, care should be taken when directly comparing with $\Delta G_{0}$ for antibody-antigen obtained with other techniques or with different set-ups: binding/unbinding free energies usually cover a range of values that are representative of the large number of different physical situations, or conformations, in which the system can appear. Here, for example, the binding free energy is obtained as a function of voltage by measuring a variation of current, governed by binding/unbinding events, which modify the local atomic charges and their position. The greater number of such events, the larger the variation of current. Yet, the current variation is mostly contributed by (un)binding stemming from conformations characterized by lower energy barriers, while those featuring higher barriers lead to fewer binding/unbinding events and contribute less to the variation of current.

In order to compare the best fit values of the electrostatic contributions with their areal density counterparts, which are of more common use in device assessement, we first estimate the projected area $\mathrm{A}_{\mathrm{Ab}}$ of an oriented antibody molecule to be about $100 \mathrm{~nm}^{2}{ }^{34}$. Then, by assuming that the coverage is $\Theta \approx 30 \%{ }^{13}$, the molar area turns out to be $A_{m o l}=N_{A} \cdot A_{A b} / \Theta=2 \cdot 10^{12}$ $\mathrm{cm}^{2} \cdot \mathrm{mol}^{-1}$, where $N_{A}$ is Avogadro's constant. By dividing $\delta Q_{m, e f f}$ by $A_{m o l}$ the areal change of the electric charge is $8.6 \mathrm{nC} \cdot \mathrm{cm}^{-2}$.

In the investigated range, the charge contribution to the electrostatic free energy is negative, ranging from -1.7 to $-3.4 \mathrm{~kJ} \mathrm{~mol}^{-1}$, as the effective change of the charge upon binding $\delta Q_{m, e f f}$ is positive. The ratio between the best fit value $17.3 \mathrm{kC} \mathrm{mol}^{-1}$ and the Faraday constant is about 0.18 , thus suggesting that only a partial charge rearrangement takes place. In fact, since TNF $\alpha$ in solution is slightly negative, we infer that most of this contribution arises from the change of dipole moment at the interface. The charge contribution is mostly important in the sub-threshold regime, i.e. for small and negative $\left(V_{\mathrm{GS}}-V_{\mathrm{th}}\right)$

The capacitive contribution to the free energy is positive, thus destabilizing, as expected by an in-series expansion of the electrostatic free energy. In the investigated range, the capacitive contribution ranges from +0.7 to $+2.8 \mathrm{~kJ} \mathrm{~mol}^{-1}$. The capacitive contribution is mostly important in the accumulation regime (i.e. for large and negative $\left.\left(V_{\mathrm{GS}}-V_{\mathrm{th}}\right)\right)$. As a result of the 
two opposing but comparable in magnitude contributions, the minimum free energy of binding $\left(\Delta G_{0}+\Delta G_{e}\right)_{\min }$ upon modulation of the gate voltage is:

(16) $\left(\Delta G_{0}+\Delta G_{e}\right)_{\min }=\Delta G_{0}-\frac{1}{4} \frac{\delta Q_{e f f}^{2}}{\delta C_{e f f}} \cong \Delta G_{0}-1.1 \mathrm{~kJ} \mathrm{~mol}^{-1}$

Albeit small, the lowering of the free energy partially compensates the effect of the thermal energy $R T$ on the binding. Tuning the gate voltage in such a way to fulfill eq.16, viz. ( $V_{G S}-$ $\left.V_{t h}\right)_{\min }=-\frac{1}{2} \frac{\delta Q_{e f f}}{\delta C_{e f f}}$, will stabilize the binding.

Similarly to what was detailed above for $\delta Q_{m, e f f}$, we divide $\delta C_{m, \text { eff }}$ by the molar area $A_{m o l}$ of the antibody to yield a change of capacitance density $\delta C_{\text {eff }}=\delta C_{m, e f f} / A_{\text {mol }} \approx 35 \mathrm{nF} \cdot \mathrm{cm}^{-2}$. This value, albeit two-three orders of magnitude smaller than the capacitance of the double layer of the electrolyte ${ }^{35}$, is comparable to the capacitance density of technology dielectrics like silicon oxide. This means that the capacitance due to the binding of the antigen to the antibody becomes dominant once in series with much larger capacitances, like the one of the double layer of the electrolyte. This is in qualitative agreement with the concept of Donnan capacitance $^{2,11}$, introduced earlier ${ }^{11}$ to justify detection in EGOFETs in the presence of recognition phenomena occurring at length scales beyond the Debye length. Our results and their interpretative theoretical framework highlight that also other electrostatic contributions in addition to capacitive coupling can modulate the device response to biorecognition.

The consistency of the data evidenced by our analysis strongly hints to the possibility to use the EGOFET biosensor as an experimental tool to extract and assess the thermodynamics of biomolecular recognition at solid/liquid interfaces and provide valuable input for understanding the detailed mechanism of biorecognition at surfaces.

\section{Selectivity and interference assays on in vitro and human samples}

The selectivity of the EGOFET was assessed by screening the response of our device against PBS solutions containing different concentrations of Bovine Serum Albumin (BSA). BSA is used as a standard interfering agent in control experiments of biosensing units on solid supports, due to its tendency to non-specifically adsorb to surfaces ${ }^{10,36}$. The dose curve $S$ vs [BSA] is shown in fig. 5a, and compared to the signal recorded for the same concentrations of TNF $\alpha$ : only minor variations of $\mathrm{S}$ can be observed upon exposure to BSA, rather negligible with respect to those recorded in the presence of TNF $\alpha$. No direct correlation between $S$ and [BSA] can be extrapolated. It is therefore reasonable to assume that the small current decrease 
is due to non specific adsorption of BSA on the gate electrode spots where no His-tag Protein $\mathrm{G}$ (PG) is present, but that the changes in the transfer characteristics as in fig.1d might still be confidently ascribed to specific binding events between TNF $\alpha$ and its surface-immobilized antibody.
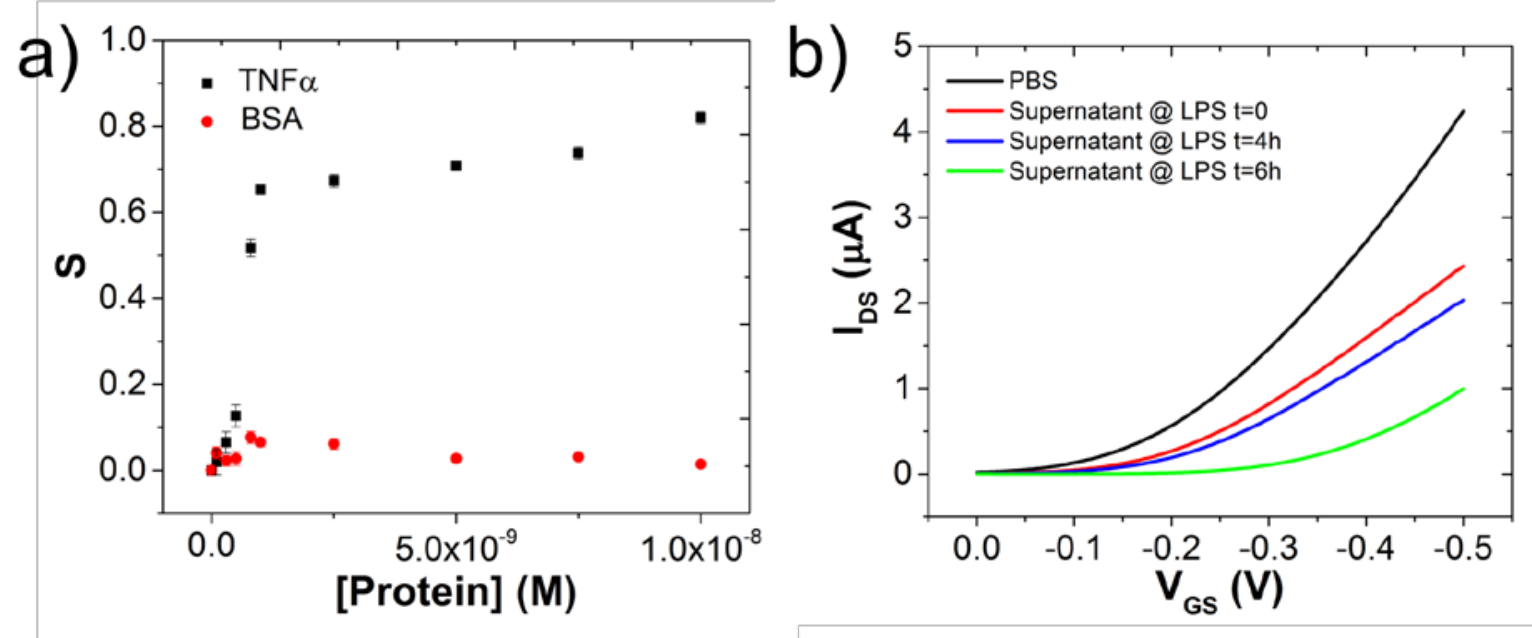

Figure 5. a) Dose curve S vs concentration of TNF $\alpha$ (black squares) and BSA (red circles) acquired at $V_{G S}=-0.8 \mathrm{~V}$. The error bars correspond to the $r$ s of $S$ averaged over 4 devices measured from two different test patterns. b) Transfer characteristics of a pentacene EGOFET upon exposure of the device to PBS (black) and to the supernatant of purified human monocytes cultured in vitro and stimulated with LPS at $t=0$ (red) and after 4 (blue) and 6 (green) hours. Please note that the transfer characteristics shown in panel b were recorded using a slightly modified setup (see Experimental section for details) .

A further proof of selectivity was obtained by assaying more complex matrices, with the aim of assessing the ability to detect human biomarkers in real samples such as plasma or serum. To this aim, human monocytes isolated by a healthy donor were cultured in-vitro in RPMI1640 complete culture medium; TNF $\alpha$ expression and release was induced by the addition of $0.1 \mu \mathrm{g} / \mathrm{mL}$ lipopolysaccharide (LPS). Supernatant aliquots were obtained just after LPS induction ( $\mathrm{t}=0)$ and after 4 and 6 hours. The monocytes culture medium is a more complex environment than PBS, as it contains high salt concentration, antibiotics, and, most notably, $10 \%$ of FBS (i.e. fetal bovine serum), which supplements the medium with vitamins, growth factors, electrolytes and several proteins. It is therefore not surprising to observe changes in the transfer characteristics (fig.5b) when switching from simple aqueous solutions to the cell culture medium. Nevertheless, significant decrease of $I_{D S}$ can be observed between the sample at $\mathrm{t}=0$ and those collected hours after induction of $\mathrm{TNF} \alpha$ production. As the only difference between these samples is the increasing quantity of the cytokine released in solution upon LPS stimulation, the changes in the transfer characteristics are due to the transduction of the selective binding of our biomarker of interest to the functionalized gate electrode. These 
results support the capability of our sensing device in unprocessed, highly complex samples as prototypes of bodily fluids.

\section{CONCLUSIONS}

In conclusion, our work has demonstrated that EGOFETs, configured as immune-sensors, can achieve ultra-sensitive detection of antigens in solutions and (models of) bodily fluids. This is due to the fact that the concentration of the antigen is correlated to the electrostatic potential change of the gate electrode, which is then "mirrored" by the shift of the Fermi level in the semiconductor. The super-exponential rise of the sensitivity parallels the energy variation of the DOS. Therefore, small changes of Fermi level in the tail of the pentacene DOS, caused by gate adsorption of ultra small quantities of the antigen, will result in large variations of the device response.

The EGOFET response was described with a combined model of recognition which effectively interpolates between two limiting regimes: Hill-type (describing the superexponential) and Langmuir-type. By means of best fit, we extracted the binding constant, the $V_{G S}$-dependence of which allows estimation of the standard Gibbs free energy change upon biorecognition. The EGOFET response also embodies the variation of the charge and the capacitance (or impedance) at the recognition interface. This provides insight into the electrostatic changes occurring upon recognition, and on the importance of subtle changes at the interface being amplified in the device response.

Finally, we demonstrated that the gate voltage is a crucial experimental handle for tuning the sensitivity of the device, which can be maximized at the lowest concentrations by operating the transistor in the sub-threshold regime, and at the highest concentrations in saturation regime.

\section{ASSOCIATED CONTENT}

Supporting Information

Further details concerning the Experimental section (transistors; reagents; protocols for gate 
functionalization; isolation of monocytes and stimulation with lipopolysaccharide). This material is available free of charge via the Internet at http://pubs.acs.org

\section{ACKNOWLEDGEMENTS}

We gratefully acknowledge IT MIUR Bilateral Project Italy/Sweden "Poincaré” for support, Italian CNR Flagship Project NANOMAX, and EU Project “iONE-FP7”. CAB and MP acknowledge support from Life Science Department through "FAR2015". CAB acknowledges the "Fondazione di Vignola" for support. 


\section{REFERENCES}

(1) Janata, J. J. Am. Chem. Soc. 1975, 97, 2914-2916.

(2) Janata, J. Anal. Chem. 1987, 59, 1351-1356.

(3) Bergveld, P. Biosens. Bioelectron. 1991, 6, 55-72.

(4) van Hal, R. E. G.; Eijkel, J. C. T.; Bergveld, P. Adv. Colloid Interface Sci. 1996, 69, 31-62.

(5) Lieber, C. M. MRS Bull. 2003, 28, 486-491.

(6) Gruner, G. Anal. Bioanal. Chem. 2005, 384, 322-335.

(7) Hammock, M. L.; Knopfmacher, O.; Naab, B. D.; Tok, J. B. H.; Bao, Z. ACS Nano 2013, 7, 3970-3980.

(8) Hammock, M. L.; Knopfmacher, O.; Ng, T. N.; Tok, J. B. H.; Bao, Z. Adv. Mater. 2014, 26, 6138-6144.

(9) Liao, C.; Mak, C.; Zhang, M.; Chan, H. L. W.; Yan, F. Adv. Mater. 2015, 676-681.

(10) Sharma, R.; Deacon, S. E.; Nowak, D.; George, S. E.; Szymonik, M. P.; Tang, A. A. S.; Tomlinson, D. C.; Davies, A. G.; McPherson, M. J.; Wälti, C. Biosens. Bioelectron. 2016, 80, 607-613.

(11) Palazzo, G.; De Tullio, D.; Magliulo, M.; Mallardi, A.; Intranuovo, F.; Mulla, M. Y.; Favia, P.; Vikholm-Lundin, I.; Torsi, L. Adv. Mater. 2015, 27, 911-916.

(12) Casalini, S.; Leonardi, F.; Cramer, T.; Biscarini, F. Org. Electron. 2013, 14, 156-163.

(13) Casalini, S.; Dumitru, A. C.; Leonardi, F.; Bortolotti, C. A.; Herruzo, E. T.; Campana, A.; de Oliveira, R. F.; Cramer, T.; Garcia, R.; Biscarini, F. ACS Nano 2015, 9, 50515062.

(14) Mulla, M. Y.; Tuccori, E.; Magliulo, M.; Lattanzi, G.; Palazzo, G.; Persaud, K.; Torsi, L. Nat. Commun. 2015, 6, 6010.

(15) Trilling, A. K.; Beekwilder, J.; Zuilhof, H. Analyst 2013, 138, 1619.

(16) Jang, M.; Kim, H.; Lee, S.; Kim, H. W.; Khedkar, J. K.; Rhee, Y. M.; Hwang, I.; Kim, K.; Oh, J. H. Adv. Funct. Mater. 2015, 25, 4882-4888.

(17) Manoli, K.; Magliulo, M.; Mulla, M. Y.; Singh, M.; Sabbatini, L.; Palazzo, G.; Torsi, L. Angew. Chemie Int. Ed. 2015, 54, 12562-12576.

(18) Duan, X.; Li, Y.; Rajan, N. K.; Routenberg, D. A.; Modis, Y.; Reed, M. A. Nat. Nanotechnol. 2012, 7, 401-407.

(19) Braden, B. C.; Dall’Acqua, W.; Eisenstein, E.; Fields, B. A.; Goldbaum, F. A.; Malchiodi, E. L.; Mariuzza, R. A.; Schwarz, F. P.; Ysern, X.; Poljak, R. J. Pharm. Acta Helv. 1995, 69, 225-230. 
(20) Gould, H. J.; Sutton, B. J.; Beavil, A. J.; Beavil, R. L.; McCloskey, N.; Coker, H. A.; Fear, D.; Smurthwaite, L. Annu. Rev. Immunol. 2003, 21, 579-628.

(21) Karlsson, R.; Michaelsson, A.; Mattsson, L. J. Immunol. Methods 1991, 145, 229-240.

(22) Landauer, R. Zeitschrift für Phys. B Condens. Matter 1987, 68, 217-228.

(23) Endres, R. G.; Fong, C. Y.; Yang, L. H.; Witte, G.; Wöll, C. Comput. Mater. Sci. 2004, 29, 362-370.

(24) Yogev, S.; Halpern, E.; Matsubara, R.; Nakamura, M.; Rosenwaks, Y. Phys. Rev. B 2011, 84, 165124.

(25) Magliulo, M.; Mallardi, A.; Gristina, R.; Ridi, F.; Sabbatini, L.; Cioffi, N.; Palazzo, G.; Torsi, L. Anal. Chem. 2013, 85, 3849-3857.

(26) Yogev, S.; Halpern, E.; Matsubara, R.; Nakamura, M.; Rosenwaks, Y. Phys. Rev. B Condens. Matter Mater. Phys. 2011, 84, 1-8.

(27) Völkel, A.; Street, R.; Knipp, D. Phys. Rev. B 2002, 66, 1-8.

(28) Puigdollers, J.; Marsal, A.; Cheylan, S.; Voz, C.; Alcubilla, R. Org. Electron. physics, Mater. Appl. 2010, 11, 1333-1337.

(29) Lee, K.; Oh, M. S.; Mun, S. J.; Lee, K. H.; Ha, T. W.; Kim, J. H.; Park, S. H. K.; Hwang, C. S.; Lee, B. H.; Sung, M. M.; Im, S. Adv. Mater. 2010, 22, 3260-3265.

(30) Sueyoshi, T.; Fukagawa, H.; Ono, M.; Kera, S.; Ueno, N. Appl. Phys. Lett. 2009, 95.

(31) Cahen, D.; Kahn, A. Adv. Mater. 2003, 15, 271-277.

(32) Gruhn, N. E.; Da Silva Filho, D. A.; Bill, T. G.; Malagoli, M.; Coropceanu, V.; Kahn, A.; Brédas, J. L. J. Am. Chem. Soc. 2002, 124, 7918-7919.

(33) Abramowitz, M.; Stegun, I. A.; Miller, D. J. Appl. Mech. 1965, 32, 239.

(34) Pease, L. F.; Elliott, J. T.; Tsai, D. H.; Zachariah, M. R.; Tarlov, M. J. Biotechnol. Bioeng. 2008, 101, 1214-1222.

(35) Cramer, T.; Kyndiah, A.; Murgia, M.; Leonardi, F.; Casalini, S.; Biscarini, F. Appl. Phys. Lett. 2012, 100, 143302.

(36) Angione, M. D.; Cotrone, S.; Magliulo, M.; Mallardi, A.; Altamura, D.; Giannini, C.; Cioffi, N.; Sabbatini, L.; Fratini, E.; Baglioni, P.; Scamarcio, G.; Palazzo, G.; Torsi, L. Proc. Natl. Acad. Sci. 2012, 109, 6429-6434. 
For TOC only

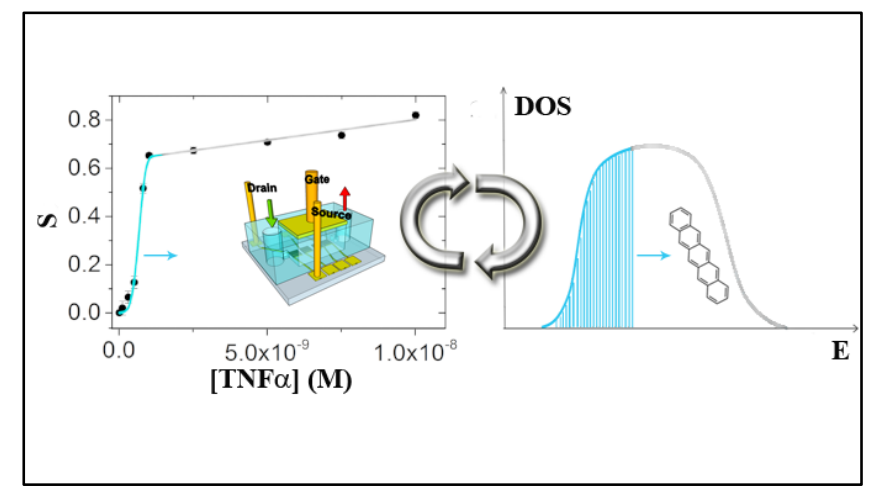

